\title{
The Physical Chemical Frontiers of Mass Independent Isotope Effects
}

\author{
M.H.THIEMENS ${ }^{1}$ \\ ${ }^{1}$ Department of Chemistry and Biochemistry, University of \\ California San Diego, La Jolla, Ca. 92093-0340. \\ 2 \\ (mthiemens@ucsd.edu)
}

Mass Independent Processes and the Future

The discovery of a chemically produced mass independent isotope effect by Thiemens and Heidenreich and application in nature dates to 1983 [1] are now applied in studies on the origin of the solar system, life, stratospheric chemistry, greenhouse gas chemistry and source recognition, paleo studies of the oxidative capacity of earth, Mars atmospheric chemistry, and quantum chemistry using sulfur and oxygen isotopes.

MIF occurs in bond formation where the isotopic selectivity does not involve conventional kinetic or thermodynamic effects. The second MIF process is photochemical and a result of isotopic selectivity associated with the actual bond formation and transition state selectivity. Recently Thiemens and Lin [2] reviewed these MIF processes and experiments, theory and computation. For combination reactions associated with producing solar nebular $\mathrm{CAI}$, atmospehric ozone and transfer of triple oxygen isotopic anomalies to $\mathrm{CO}_{2}, \mathrm{O}_{2}, \mathrm{~N}_{2} \mathrm{O}$, $\mathrm{CO}, \mathrm{SO}_{4}$, and $\mathrm{NO}_{3}$ loose transition state approaches with quantum coupling to exit channels dependent upon density of states,isotopic symmtery, zero point energy differences, and hindered rotations have been developed (Marcus) that define anomaly production. Babikov approaches the formation step using quantum computational 3-D potential energy surfaces via metastable state energy transfers. Present day atmopsheric sulfur reactions and interpreting Archean observations by sulfur reactions electronically analagous to ozone formation [3] consistent with early experiments at UCSD [1] require inclusion of these effects. These reactions need deeper mechanistic understanding to interpret MIF sulfur isotope records. For photodissociation and Archean sulfur isotopic anomalies there are limits to mechanistic theory and experiments of the isotope effect in photodissociation as a function of wavelngth. Advancement is needed for the next generation of geochemical models.

[1] Thiemens \& Heidenreich (1983) Science 219, 1073-1075. [2] Thiemens and Mang (2019) Angewandte Chemie 58, 68266844. [3] Babikov (2017). Proc Nat. Acad. Sci 114, 3062-3067. 